\title{
La campaña electoral como ritual de acceso al poder
}

\section{The electoral campaign as a ritual of access to power}

\author{
María de la Serna Ramos ${ }^{1}$ \\ Ministerio de Industria, Comercio y Turismo \\ mariadlserna@gmail.com
}

Recepción: 18/12/2018 Revisión: 12/03/2019 Aceptación: 19/05/2019 Publicación: 01/06/2019

\begin{abstract}
Resumen
Las campañas electorales son el paso previo al acceso al poder institucional de los políticos. Durante los quince días previos a las votaciones se llevan a cabo multitud de eventos de diferentes características que buscan presentar al candidato imbuido en su papel de futuro gobernante: son actos de puesta en escena de sus valores, formas y habilidades. No se diseñan sólo para transmitir un programa, sino, que por encima de todo, lo que pretenden es poner al "futurible" en un entorno propicio para emitir un mensaje de poder. La complejidad de aunar en una buena producción, un buen escenario y un buen mensaje personalizado en el candidato requiere del trabajo en equipo de muchos especialistas: asesores de imagen, escenógrafos y técnicos de protocolo son fundamentales para poner en pie una ilusión que lleve al votante a depositar un determinado voto en las urnas. La complejidad de estos actos, sean de la naturaleza que sean, conlleva un trabajo previo y de un desarrollo en el que el protocolo juega un papel fundamental. En el momento que hay un líder, hay un protocolo.
\end{abstract}

Palabras claves: Campaña electoral, eventos, poder, políticos.

\section{Abstract}

Election campaigns are the previous step for politicians to access institutional power. The numerous events of different kinds held fifteen days prior to voting seek to present the future ruler's values, manners and skills. Their design is not only meant to present a program, most

1 Técnico en Protocolo y Relaciones Institucionales por la Escuela Internacional de Protocolo y Graduado Universitario en Protocolo y Relaciones Institucionales por la Universidad de Granada; socia fundadora de la empresa GMS Protocolo y Eventos, administradora del blog Protocolo a la Vista, jefa de Protocolo del Ministerio de Industria, Comercio y Turismo. 
of the times briefly reviewed before the voters, but to create an enabling environment for sending out a message of power: he is the leader, he has the power and exercises it.

The complexity of these acts, which requires a previous teamwork of different specialists like image consultants, set designers and protocol technicians are the main tool to stir up the excitement leading voters to cast ballots in polling stations. In the development of these events, the protocol plays a key role.

We'll discuss a few examples of different events, their production and development, as well as all agents involved in them, from the very first idea to the launch of the event.

Keywords: elections campaings, events, power, politicians

\section{Sumario}

1. Introducción

1.1. Hipótesis y objetivo

1.2. Metodología

2. La campaña electoral como ritual de acceso al poder

2.1. Los primeros años: 1977 a 1982

\subsubsection{Primeras campañas}

2.1.2. Primeros mítines y apariciones públicas

3. La última década: 2004 a 2015

3.1. Las estrategias

3.2 Las puestas en escena

4. Conclusiones

\section{INTRODUCCIÓN}

Las campañas electorales se han ido sofisticando con el tiempo. Desde las primeras de los años 1977 y 1979, en las que primaba el ansia por votar, a las últimas de diciembre de 2015 , en las que lo primordial era "vender" al candidato, el concepto de "campaña electoral" ha evolucionado a la vez que lo hacía la sociedad española adaptándose con rapidez y muchas veces con antelación, a las nuevas formas de comunicación, a los medios de comunicación y a la percepción que sobre la política tienen los ciudadanos. 
Los años de democracia, las expectativas de los ciudadanos, el desencanto que el tiempo provoca en los votantes y, especialmente, su alejamiento de la política y de los políticos, el hastío y el poco interés por los partidos que les han provocado la desilusión ha obligado a éstos a modificar drásticamente su estrategia. Giramos cada vez más hacia un tipo de campañas del estilo de las norteamericanas: la mercadotecnia al servicio de las formaciones políticas. Especialmente al servicio de un candidato que es, a su vez, la opción que los aparatos de los partidos políticos consideran que es la que reúne las condiciones necesarias para alcanzar la presidencia. Son "figuras volubles y marionetas versátiles al servicio de los asesores" y que en ellos "unos especialistas (...) ven (...) al candidato como un producto de mercado dispuesto y preparado para ser vendido al mejor postor ${ }^{2 \prime}$. El valor intrínseco de la persona, su capacidad, preparación y habilidad no son determinantes.

\subsection{Hipótesis y objetivo}

Partiendo de esta idea, el objetivo del presente trabajo es poner de relieve que esta "venta" del candidato pasa por mostrarle u ofertarle en sus apariciones públicas con el perfil que la sociedad actual busca en un presidente de gobierno. Siguiendo guiones diseñados por publicistas y apoyándose en encuestas, estadísticas y estudios sociológicos, los asesores bucean en las preferencias de los votantes y dotan a sus candidatos de las condiciones que han comprobado que éstos prefieren. Para que pueda apreciarse el ajuste del candidato a las preferencias de los posibles votantes, todos los actos a los que acuden -debates, entrevistas, apariciones en televisión, campañas electorales, ruedas de prensa...- no se dejan al azar y se utilizan como plataformas o escaparates de esas virtudes solicitadas. Son parte de la estrategia y como tal tienen un objetivo final. Errar en la estrategia de venta, es perder la presidencia del gobierno.

\subsection{Metodología}

La metodología de trabajo se apoya básicamente en el método cualitativo: indirectas (bibliografía, revistas, internet, fotografías de la época) y directas (lectura de dosieres y documentación sobre campañas de los partidos políticos).

Para poder llegar a conclusiones se han analizado las puestas en escena de las apariciones públicas de los políticos españoles más relevantes desde la llegada de la democracia en las campañas a generales celebradas desde entonces, dividiéndolas en dos bloques: 1977 a 1982 -primeras campañas electorales y mítines- y 2004 a 2015 -campañas electorales, debates y mítines. Se han estudiado los escenarios pero también los contenidos

\footnotetext{
${ }^{2}$ SANTIAGO BARNÉS, J (2007): El político nace o se hace. Salamanca. Amarú Ediciones
} 
creados para los mítines, así como la apariencia de los protagonistas, como portadores de los mensajes de sus respectivos partidos.

\section{LA CAMPAÑA ELECTORAL COMO RITUAL DE ACCESO AL PODER}

\subsection{Los primeros años: 1977 a 1982}

Los políticos españoles que se lanzaron al ruedo tras la dictadura no tenían experiencia alguna ni en campañas electorales, ni en disciplinas que les dieran cierta ventaja para competir contra otras opciones. En aquellos primeros años su gran preocupación era que los ciudadanos no fuesen a votar. Y por ello en toda la cartelería de la época aparece la palabra "vota", además del resto de elementos que debe contener -foto, logotipo y lema- y que en cada caso resumían y resaltaban las diferencias entre las opciones que ofertaban los diferentes partidos políticos.

El candidato de hace cuarenta años se desgañitaba ante sus seguidores volcando sobre el público todo su programa para que éste pudiera valorar la diferencia que había entre una u otra opción política. Ni su atuendo, ni el espacio elegido para dar un mitin tenían especial relevancia: simplemente se buscaba un recinto lo suficientemente amplio para que cupiese la audiencia estimada. La producción de los actos era artesanal: los fondos se pintaban a mano, no había elementos decorativos, y salvo mesas tapadas con carteles o cualquier tipo de tapetes, nada había que distrajese la atención. Se centraba la acción en el orador, sin apenas escenografía y sin protocolo.

La televisión era el medio habitual a través del que se informaba la mayoría de los ciudadanos. No había televisiones privadas ${ }^{3}$ y era RTVE la que marcaba pautas, calendario y posibilidades de copar pantalla.

\subsubsection{Primeras campañas}

Las primeras elecciones generales en España se celebraron el 15 de junio de 1977. Pero no fue la campaña electoral diseñada por los diferentes partidos políticos la primera que vivieran los españoles. En diciembre de 1976 se había votado en referéndum la aprobación, o no, de la Ley para la Reforma Política. En la campaña correspondiente en busca de la participación de los votantes, el uso de los medios de comunicación fue muy grande. El entonces presidente Adolfo Suárez había sido director general de Radiotelevisión Española y era muy consciente del poder que el medio televisivo tenía y lo muy útil que resultaba para trasladar mensajes.

${ }^{3}$ El 26 de agosto de 1989 se concedió licencia a tres empresas para empezar a emitir a partir de abril de 1990: Antena 3 de Televisión, S.A., Gestevisión Telecinco, S.A., y Sogecable, S.A. 
El 10 de septiembre de 1976, el presidente Suárez presentó en un discurso por televisión el texto del Proyecto de Ley para la Reforma Política. Es

"un ejemplo evidente de cómo el presidente utilizó la televisión para lograr crear una opinión favorable a la ley, que culminaría con otro discurso pronunciado antes de la celebración del referéndum. Imagen en color, el programa informativo "Habla el presidente del gobierno» ya se inicia con una sintonía musical más alegre y pegadiza. La retransmisión se inicia con el entrando en la sala camino de su mesa y saludando a los ministros que le acompañan en la sala. Imagen dinámica y próxima, en un decorado sencillo donde predomina la fuerza del blanco"."

Apoyándose así en la televisión, se lanzó una campaña sencilla pero muy eficaz para atraer a los votantes a las urnas. El gobierno la controló a través de la Dirección General de RTVE -a cuya frente estaba Rafael Ansón, especialista en comunicación-. Además, se contrató publicidad creada al efecto por una serie de agencias para promover la participación de los españoles.

De esta primera campaña, en la que no se decidía candidato, y en la que se buscaba además de la asistencia masiva a las urnas, obtener un "si" a la reforma política, son lemas tan populares como Habla, pueblo -canción del grupo murciano Vino Tinto y cuyos derechos compró la UCD, el partido de Adolfo Suárez-, Infórmate bien, y vota o Tu voz es tu voto. Mensajes sencillos que incitaban a votar y a votar afirmativamente.

En las campañas posteriores de 1977, 1979 y 1982, la figura del candidato ocupó ya todos los espacios disponibles. Los partidos crearon equipos para elaborar estrategias determinadas a obtener la mayoría de los votos.

El gobierno concedió espacios gratuitos ${ }^{5}$ en los medios bajo su control -televisión, radio y prensa- a todas las formaciones que se presentasen al menos en 25 circunscripciones y se estableció una franquicia postal especial para el envío de propaganda electoral.

Basándose en ideas-clave, que plasmaron en sus carteles electorales, los equipos electorales de los partidos diseñaron en 1977 sus respectivas campañas para que el electorado les identificase con el camino por el que querían transitar. Las tres principales formaciones

\footnotetext{
${ }^{4}$ http://www.publicacions.ub.es/bibliotecadigital/cinema/filmhistoria/Art.Ardanaz.pdf

${ }^{5}$ Decreto $967 / 77$ de 3 de mayo
} 
trabajaron a partir de los conceptos "libertad" (PSOE), "seguridad" (UCD), "España" (AP) y "no somos el coco" (PC), que acababa de ser legalizado y buscaba, además, cambiar la percepción que la sociedad española tenía del partido. Aunque la principal preocupación de todos fuera que se acudiera a votar y por eso "Vota" aparece en todos los carteles.

En 1979, se pasó del abstracto de las buenas intenciones, a publicitarse como solución a los problemas concretos de los españoles. Las ideas-clave alrededor de las que jugaron los partidos eran más concretas, definidas y con un contenido preciso que reflejaba la opción política: "Un gobierno firme" (PSOE), "Dicho y hecho" (UCD) "Pon tu voto a trabajar" (PC).

Las siguientes elecciones Ilegaron muy pronto. El gobierno de Calvo Sotelo, presidente tras la moción de censura a Suárez e investido tras el fallido golpe de estado de febrero de 1981, las convocó anticipadamente para el mes de octubre de 1982. Todos los partidos afinaron sus mensajes. La situación económica, el paro y la inflación marcarán las líneas de trabajo: cada uno le dará el sesgo correspondiente a su ideología. EI PSOE prometió sus famosos 800.000 puestos de trabajo y para ello indicó que el camino era la lucha contra el fraude fiscal, el aumento de los impuestos a los que más ganaban, la mejor de las pensiones y la promoción de empresas públicas. AP-PDP, por su parte, ofreció el saneamiento de la economía, la reducción del déficit, el control de la inflación y puso el énfasis en la actividad privada como motor de la economía, que era lo mismo que ofertaba UCD, que sumaba al lote de ofertas la reconversión industrial y los incentivos fiscales a la creación de empleo. El PC lo crearía, decía, a través de una mayor actividad del sector público y de una reforma agraria: un millón de puestos de trabajo.

La campaña duró 21 días. Sin limitación de gasto electoral y con un muy deficiente control de este. Los partidos políticos invirtieron masivamente en publicidad: el PSOE contrató 5.000 vallas publicitarias, 8.000 cabinas telefónicas, una tirada de medio millón de programas, 10.000 trípticos y 32.000 cuñas radiofónicas. Las cifras de AP-PDP y UCD fueron muy similares ${ }^{6}$.

\subsubsection{Primeros mítines y apariciones públicas}

En el año 1977 se celebraron 22.000 mítines durante los 21 días de campaña : los mayores y más concurridos fueron los del PSOE y PCE que llenaron polideportivos y plazas de toros. Pero, la maquinaria propagandística puesta en marcha desde el gobierno de Suárez no

\footnotetext{
${ }^{6}$ WERT, J.I. (1984): La campaña electoral de octubre de 1982: el camino del cambio, Revista REIS, no 28, pp.75-76

7 MARTínEZ, J. (1998). Historia de España. Siglo XX (1939-1996). Madrid: Cátedra. pp. 280
} 
dejó resquicio y su presencia constante y aplastante en los medios que estaban bajo su control fue su mejor estrategia: incluso le permitió no tener que debatir con rival alguno ${ }^{8}$.

En 1979 el Ministerio de Cultura prohibió el alquiler de las instalaciones polideportivas dependientes de su departamento para la celebración de mítines u otros actos electorales durante la campaña. Por ello se plantearon los actos en recintos cerrados y locales pequeños, entre ellos cines y teatros, y se trató de evitar concentraciones masivas: los candidatos explicaron su programa en coloquios, reuniones en fábricas y centros de trabajo, mesas redondas o conferencias. Aunque las plazas de toros fueron una opción muy valorada.

Los escenarios en los mítines eran sencillos. En los recintos grandes se marcaron espacios presidenciales sencillos en tarimas, habitualmente con mesas académicas muy largas, tapadas con telas o carteles electorales de la propia formación y en las que no faltaron vasos y botellas de agua, micrófonos y material de papelería; o se utilizaron balcones, y salvo el sonido y la iluminación que requirieron de profesionales, aunque no fueron montajes complicados, el resto del escenario no era complejo. Reflejaban la necesidad de adecuar un espacio a un uso determinado -el mitin- y el único mensaje visual a emitir desde allí era la identidad corporativa del partido identificable con su logotipo: grandes cartelones, paneles en las traseras y carteles electorales pegados sobre ellos -muchas veces sujetos, incluso, con cinta adhesiva- y elaboración manual, si era necesario, recortando letras. Se utilizaron atriles cubiertos con telas como soporte de logotipos. El conjunto tenía un aspecto más artesanal que profesional. En los partidos de derecha y extrema derecha era frecuente el uso banderas cubriendo las mesas y centros de flores para decorar.

En todos los mítines jugó especial importancia la música: el jingle de UCD -“Vota centro, vota Suárez, vota libertad", el himno de $\mathrm{AP}^{10} \mathrm{O}$ "La internacional", que el PC tuneó con ayuda de un órgano Hammond, competían con el grupo Jarcha cuya canción "Libertad sin ira", escrita con motivo de la salida del periódico Diario 16 un año antes, acabó siendo el himno de la transición.

El atuendo de los candidatos no fue cuestión a tener en cuenta, y salvo que fuesen a algún programa de la televisión, ni se peinaban, maquillaban o vestían para la ocasión. Era frecuente que se les viera con el cigarrillo en la mano. Los candidatos de los partidos de centro y centro derecha vestían el habitual traje y corbata y los de la izquierda, airearon la pana, las camisas de cuadros y las mangas de camisa.

\footnotetext{
8 PRESTON, P. (2003). Juan Carlos, el rey de un pueblo (volumen II). Hospitalet: ABC, S.L. pp. 408-410

${ }^{9}$ De Juan Pardo

${ }^{10}$ De Jaime Morey
} 
Las apariciones en televisión no eran frecuentes, salvo en los espacios gratuitos establecidos por decreto. Felipe González se presentó a los españoles en una entrevista emitida en diciembre de 1976 y el presidente Suárez compareció, además, en enero de 1977 dada la situación de extrema gravedad del país.

Los candidatos se movieron por el territorio en avionetas: tanto Suárez como Felipe González las utilizaron con frecuencia para recorrer las distancias mayores y dejaron los automóviles para recorridos cortos y desplazamientos entre pueblos cercanos. Ambos se patearon los pueblos y los mítines se celebraron casi en cualquier sitio. Felipe González llegó a dar uno en Albacete encaramado a un carro y utilizando un tractor a modo de atril. Por su parte, Adolfo Suárez no se prodigó en exceso disculpando la poca participación por sus obligaciones como presidente y su presencia no sería ni constante, ni diaria.

Aunque en los escenarios se siguieron utilizando las mesas presidenciales de tipo académico, a principios de la década de 1980 se empezaron a diferenciar espacios con estructuras metálicas para realzar a los oradores.

En 1982 el PSOE organizó más de 40 mítines multitudinarios. Felipe González recorrió el país en un autobús. En muchos pueblos los mítines los daba micrófono en mano, y aunque aparentemente pudieran parecer mal organizados, estaban diseñados para que no se descontrolasen y se pudiera mantener una distancia entre la masa y el líder. En general, se presentaba al candidato Gonzáles como una persona cercana y por ello era frecuente la entrega de un ramo de rosas al final del acto, mostrando así al hombre en una situación emotiva, lo que provocaba la empatía del público.

La música cobró importancia como identificadora del partido. Julio Mengod compuso la música ("Aria de la Rosa") que sonaría en anuncios, vídeos y mítines del partido socialista para estas elecciones y desde entonces, y con diferentes arreglos, ha sido su sintonía electoral (salvo en el año 2000 en que se cambió por una diferente). Pero el mitin de cierre de campaña no se recordará por ella. En la explanada delante de la facultad de Biológicas de la Universidad Complutense se concentraron medio millón de personas ${ }^{11}$. Un minuto antes de las doce de la noche, Felipe González cerró el acto dirigiéndose a la audiencia con un lema: "el futuro es nuestro". Inmediatamente después se apagaron las luces. Los jóvenes allí concentrados encendieron sus mecheros y cantaron al unísono el Himno a la Libertad de Miguel Ríos. Se instalaba así en las campañas electorales el diseño emotivo.

11 EL PAIS, portada 27 de octubre de 1982 


\section{LA ÚLTIMA DÉCADA: 2004 A 2015}

El desarrollo del marketing político en estos últimos diez años ha sido enorme. Hoy, el candidato es pura imagen: es la cara visible de un grupo político, que se queda en segundo plano, y en la que se concentran las preferencias conocidas de los votantes.

Para que así lo refleje, un enorme equipo de profesionales en asesoría, marketing y comunicación política se esfuerza en hacer de la figura del candidato un producto más de consumo -y de consumo de masas... de votantes-: (le convierte) "en un actor que ensaye el papel de los investigadores sociales -auténticos ingenieros de emociones- han preparado para él'12".

Y si hablamos de emociones, hablamos de personas. Es decir, ante todo debe "parecer" un ser humano, con sentimientos, reacciones que provoquen empatía, cercano. Se le prepara para las apariciones públicas como un deportista que va a competir. Desde su aspecto exterior, para el que contará con asesoramiento de todo tipo, desde sastrería a peluquería, a sus gestos y movimientos, nada es casual ni se deja al azar.

Tampoco lo es el escenario. El diseño de las escenografías ya no es una mera cuestión de técnicos. Se elaboran a partir de una estrategia de comunicación en la que los mensajes y la forma de emitirlos se tienen que reflejar en todos los elementos. El objetivo final es "envolver" al candidato en aquellos símbolos, colores y eslóganes que le identifiquen con un partido y un programa de forma que el asistente al mitin, el espectador que lo vea a través de algún medio de comunicación identifique un producto concreto. Un "vendible" que contenga en todos sus componentes aquello que la sociedad reconoce como la suma de valores, formas, actitudes, necesidades o gustos aceptables e ideales. Se trata de acercarles a los diferentes grupos de personas. Lo fundamental es que se "vea" al candidato: que se reconozcan en él, en su imagen y en sus gestos, los rasgos de un presidente de gobierno.

El programa político no es más que un documento que incluso puede no presentarse al ciudadano hasta el último minuto, como ha sucedido ya en algunos casos en estas últimas campañas electorales. Pero a él se refieren constantemente los líderes de las formaciones, aunque no está, ni ha estado tampoco, como reconocen algunos políticos, pensado o escrito para que sirva de guía que lleve a una determinada opción política: "los programas electorales -como elemento de formalización de la oferta política- tienen en el contexto electoral español una reducida influencia directa en la determinación del voto. Dicho de modo más llano, nadie

12 DEL REY MORATÓ, Javier (1989): La comunicación política. Madrid. Eudema, pp. 109 
los lee. Estructuralmente están concebidos para no ser leídos $(. . .)^{13 \prime \prime}$. Tiene que haberlo, pues, de momento, es parte del juego, pero, como otros elementos del ritual de una campaña electoral, es susceptible de variaciones según las necesidades: esas que se derivan de las constantes encuestas.

\subsection{Las estratégias}

Los equipos de campaña diseñan las estrategias con técnicas de marketing y publicidad y de la mano de sociólogos. Los eslóganes cuentan y serán de nuevo reflejo de la oferta de cada partido, pero a ello habrá que sumar al candidato por encima de todo. "Vender programa" es más complejo que "vender candidato". Ya desde 1986 los eslóganes difundían mensajes menos específicos y más positivos, alegres y animosos: "iPalabra!", "Capaces de hacerlo", "Ahora", "Merecemos una España mejor" o "Juntos vamos a más".

En las Guías de Campaña que preparan los partidos se analizan las estrategias de los otros partidos competidores para poder preparar al candidato contra sus posibles ataques y dotarle de las herramientas para defenderse. En todas se habla de "objetivos" siendo "ganar las elecciones" la meta ansiada: se matiza con "obtener un resultado que permita formar gobierno" o con "ser el partido más votado". Evidentemente, "para sacar adelante sus proyectos", "cambiar el clima de crispación", "el compromiso", "el cambio" ... volviendo así a lo abstracto frente a propuestas concretas que, aunque explicarán en sus apariciones públicas, saben que enganchan menos que los mensajes emotivos: los "valores".son protagonistas.

\subsection{Las puestas en escena}

La oferta para "mostrar" al candidato es cada vez más amplia. De los mítines de los primeros años como la primera fuente de conocimiento del candidato y sus propuestas, se pasará a buscar el formato más adecuado a la persona. En la paleta de posibilidades entran los debates, las entrevistas y la participación de los candidatos en programas habituales de las televisiones, además de los mítines. Para cada una de estos tipos y para cada una de las apariciones públicas del candidato se preparará un guion concreto en el que se irán mezclando los elementos necesarios para ensalzar su figura. A la vez se les preparará para contestar correctamente, $\mathrm{o}$, para ser más exactos, como se espera que lo haga, a las cuestiones que se le van a plantear.

Los equipos de campaña negociarán hasta el detalle más pequeño: posición en los platós, colores de traseras, minutos de participación, bloques de temas a contestar, hora y

13 WERT, J.I. (año): La campaña electoral de octubre de 1982: el camino del cambio. Revista REIS, no28.

El autor lo justifica por la extensión de los mismos. Aporta para ello el número de páginas de los programas de PSOE (47), AP-PDP (189), UCD (264), PCE (48) o CDS (79). WERT, J.I. (1984): La campaña electoral de octubre de 1982: el camino del cambio, Revista REIS, no 28, pp. 69-70 
minuto de llegada a las televisiones... Se prepararán "argumentarios" a diario: se controla cualquier aparición del candidato, cualquier declaración que pueda hacer y no se puede dejar resquicio alguno al azar. La improvisación es el enemigo público.

La presentación de las campañas es el primer acto público. No del candidato, sino en nombre del candidato. Los directores de campaña y/o de comunicación utilizarán las ruedas de prensa para empezar a mostrar el "producto candidato". Su conexión con la oferta que representa y la forma de resumirlo en los lemas de campaña se explica de forma pedagógica: esto denota que no tienen muy claro que el posible votante sepa interpretar lo que se le presenta y consecuentemente, que lo que van a mirar no es tanto el contenido como el contenedor.

En la política española no hay tradición de debates y hasta la fecha no se han celebrado más que en seis ocasiones. En estas últimas elecciones de 2015 la paranoia por el control de espacio y los minutos en el plató, el orden de intervención y la indumentaria de los candidatos alcanzó cotas increíbles. Antecedentes había. En 1993, cuando se celebró el primer debate en Antena3, los equipos de campaña negociaron los temas a tratar y el orden de los turnos: hasta minutos antes del comienzo de la emisión estuvieron discutiendo por el desacuerdo sobre quién debía intervenir en el último turno. Los candidatos permanecieron sentados ante mesas enfrentadas, formato que impuso el PP, porque quería evitar comparaciones sobre la altura de Aznar con respecto a la de González.

En 2008 -hubo que esperar 15 años para esta segunda ronda- los equipos de campaña acordaron celebrar dos debates de 90 minutos que organizaría la Academia de Televisión. Se pactó prácticamente todo: los tiempos, la realización, el tipo de planos, las llegadas de los líderes e incluso, la posición de los relojes cronómetros que se instalaron en el plató. No se dejó nada al azar. Los dos candidatos protagonistas se dejaron asesorar por equipos muy numerosos de especialistas en imagen, comunicación y lenguaje no verbal.

En 2011 fue, de nuevo la Academia de Televisión quien organizó el debate. Aunque se flexibilizaron las condiciones, vista la rigidez del formato previo, la negociación fue dura, pero el resultado del conjunto, similar.

En las elecciones de 2015, los partidos tuvieron que acabar aceptando que sin debates las campañas cojeaban. Con el desarrollo alcanzado en especialidades tales como comunicación o marketing político, estos formatos podían ofrecer -bien planificados y trabajados- una oportunidad de oro para sus candidatos. Eran un buen escaparate. Se celebraron tres: un "cara a cara" organizado por el diario "El País", un debate a cuatro en AtresMedia y el habitual entre los principales candidatos organizado por la Academia de Televisión.

Se prepararon al detalle. Sirvan de ejemplo la descripción de la escenografía del plató de ATresMedia, el cronograma del sorteo de puestos y turnos de intervención de los cuatro 
participantes en este debate, la escaleta o las especificaciones técnicas de los atriles utilizados en el cara a cara de El País.

\section{MEMORIA}

\section{DECORADO}

-El decorado carece de colores dominantes. La luz aportará dinamismo y profundidad.

-En los planos cortos, los fondos quedarán desenfocados, buscando el protagonismo en el candidato.

-Cada candidato dispondrá de un bloque accesorio en el que podrá guardar sus documentos personales y

donde podrá tener fácil acceso a un vaso de agua.

-El área de intervención de cada candidato, está delimitada por un círculo en el suelo.

\section{ILUMINACION}

-La luz es suave, evitando grandes contrastes. Está pensada para favorecer tanto en planos generales, como en medios y cortos.

\section{PÚBLICO}

-El público solo tendrá presencia en los planos de los presentadores, nunca en los planos de los candidatos.

\section{ESTILISMO}

- Los candidatos podrán usar cualquier color en su ropa, dadas las características neutras del decorado.

-Deberán tener en cuenta que se les verá de cuerpo entero y evitar prendas brillantes o que puedan producir moaré (estampados con motivo pequeño o rayas finas).

Fig. 1. Escenografía Debate AtresMedia

Fuente: PSOE, material de campaña 


\begin{tabular}{|l|l|l|}
\hline Hora & Encargado & Acción \\
\hline $\begin{array}{l}10.00 \mathrm{a} \\
10.30 \mathrm{~h} .\end{array}$ & $\begin{array}{l}\text { llegada de representantes de los partidos a } \\
\text { instalaciones de Atresmedia }\end{array}$ & Entrada en maquillaje \\
\hline $10.30 \mathrm{~h}$ & & Reproducir recorrido del 7D y mostrar escenografía \\
\hline $11.15 \mathrm{~h}$ & & Microfonado y ensayo del sorteo \\
\hline $11.45 \mathrm{~h}$. & $\begin{array}{l}\text { Con representantes de los cuatro partidos: portavoz } \\
\text { del comité electoral del PSOE, jefa de gabinete de } \\
\text { la vicepresidenta del gobierno, secretario de } \\
\text { comunicación de Ciudadanos y la coordinadora del } \\
\text { equipo de Podemos }\end{array}$ & $\begin{array}{l}\text { Triple sorteo en directo retransmitido por Espejo Público y } \\
\text { Al rojo vivo simultáneamente. } \\
\text { Pastortado por los moderadores Vicente Vallés y Ana }\end{array}$ \\
\hline $11.45 \mathrm{~h}$. & & $\begin{array}{l}\text { Los moderadores entran al plató 5 y desde su posición en } \\
\text { el set dan la bienvenida a las cuatro personas asignadas por } \\
\text { los partidos }\end{array}$ \\
\hline $11.46 \mathrm{~h}$. & & $\begin{array}{l}\text { Los representantes se colocan detrás de tres contenedores } \\
\text { transparentes con cuatro bolas cada uno, en cuyo interior } \\
\text { están los números 1,2,3 y 4. }\end{array}$ \\
\hline $11.47 \mathrm{~h}$. & & $\begin{array}{l}\text { Primer sorteo para determinar la ubicación de los } \\
\text { participantes del debate en el plató }\end{array}$ \\
\hline $11.49 \mathrm{~h}$. & & $\begin{array}{l}\text { Segundo sorteo para determinar el orden de la intervención } \\
\text { inicial }\end{array}$ \\
\hline $11.51 \mathrm{~h}$. & & $\begin{array}{l}\text { Tercer sorteo para determinar el orden de la intervención } \\
\text { final }\end{array}$ \\
\hline $11.53 \mathrm{~h}$. & & $\begin{array}{l}\text { Ana Pastor y Vicente Vallés emplazan a los espectadores } \\
\text { al 7D: día del debate decisivo }\end{array}$ \\
\hline $11.54 \mathrm{~h}$. & & Final sorteo \\
\hline
\end{tabular}

Fig 2. Cronograma del sorteo celebrado el 3 de diciembre 2015 para el debate en Antena 3

(Fuente: PSOE, material de campaña) 
ATRILES

Trasera

Frontal

Lateral
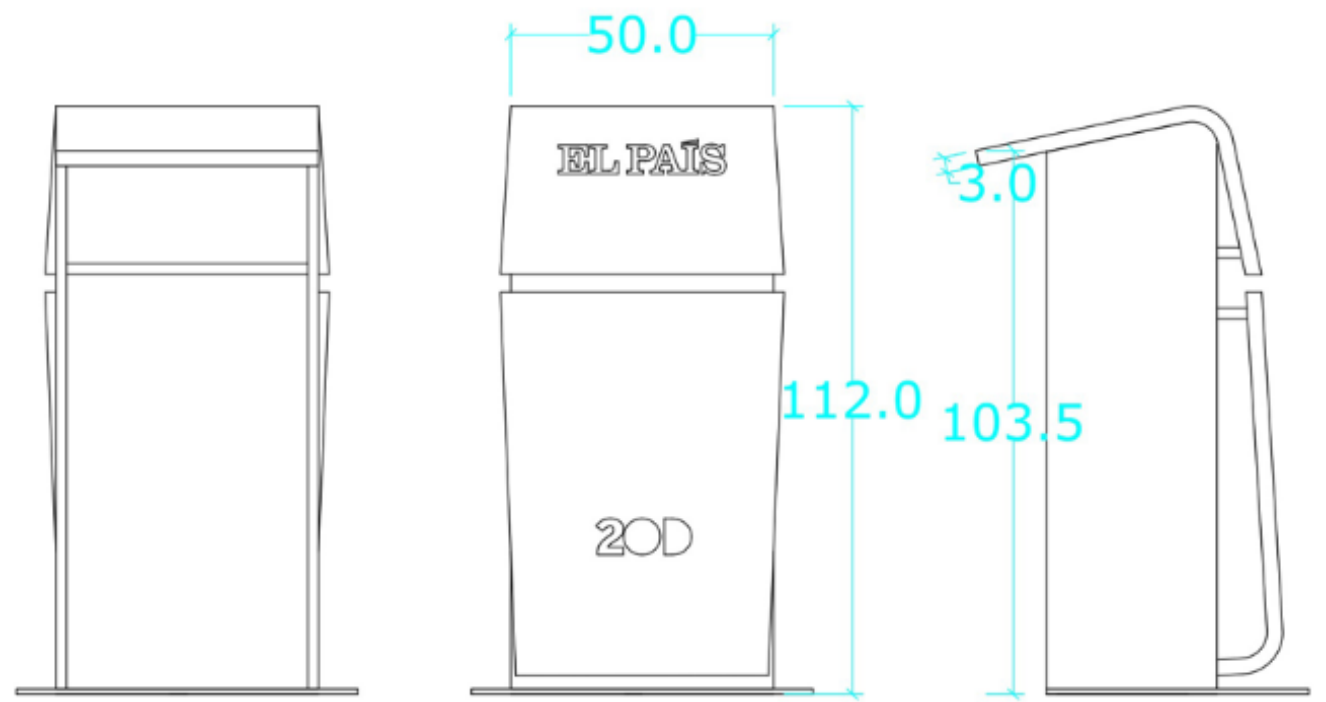

Fig. 3. Atril del "cara a cara" de El País

Fuente: PSOE, material de campaña 


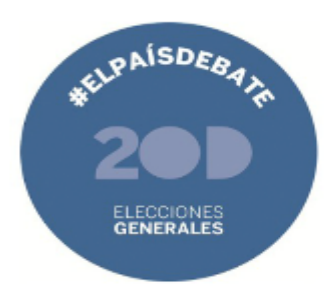

\section{EL PAÍS DEBATE 20D V4}

\begin{tabular}{|c|c|c|c|c|c|}
\hline $\mathrm{N}^{\mathrm{a}}$ & $\begin{array}{l}\text { HORA } \\
\text { INICIO } \\
\text { Bloque }\end{array}$ & CONCEPTO & $\begin{array}{c}\text { TIEM } \\
\text { PO } \\
\text { PARC } \\
\text {. }\end{array}$ & $\begin{array}{c}\text { HORA } \\
\text { FIN } \\
\text { bloque }\end{array}$ & OBSERVACIONES \\
\hline 1 & $\begin{array}{l}21 \mathrm{~h} 00 \\
00 "\end{array}$ & VTR llegada candidatos & $40 "$ & $\begin{array}{l}21 \mathrm{~h} 00 \\
40 "\end{array}$ & \\
\hline 2 & $\begin{array}{l}21 \mathrm{~h} 00 \\
40 "\end{array}$ & $\begin{array}{l}\text { CABECERA EL PAÍS } \\
\text { DEBATE }\end{array}$ & $10 "$ & $\begin{array}{c}21 \mathrm{~h} \\
00^{\prime} 50^{\prime \prime}\end{array}$ & \\
\hline 3 & \begin{tabular}{|l|}
$21 \mathrm{~h} 00^{\prime}$ \\
$50^{\prime \prime}$ \\
\end{tabular} & Saludo Carlos de Vega & $2^{\prime}$ & $\begin{array}{c}21 \mathrm{~h} \\
02 ' 50 \text { ", }\end{array}$ & \\
\hline 4 & $\begin{array}{l}21 \mathrm{~h} 02^{\prime} \\
50^{\prime \prime}\end{array}$ & $\begin{array}{l}\text { Tema Internacional + } \\
\text { Pregunta }\end{array}$ & 5 , & $\begin{array}{l}21 \mathrm{~h} \\
07 \text { '50", }\end{array}$ & $\begin{array}{l}\text { 1' cada uno. Albert, } \\
\text { Pedro, Iglesias }\end{array}$ \\
\hline 5 & $\begin{array}{l}21 \text { h } 07 \\
50 \text { '” }\end{array}$ & $\begin{array}{l}\text { PRIMER BLOQUE } \\
\text { Economía y Empleo }\end{array}$ & $30 "$ & $\begin{array}{c}21 \mathrm{~h} \\
08^{\prime} 20^{\prime \prime}\end{array}$ & Entra rótulo bloque \\
\hline 6 & \begin{tabular}{|l|}
$21 \mathrm{~h}$ \\
$08^{\prime} 20^{\prime \prime}$
\end{tabular} & $\begin{array}{l}\text { Presentación propuestas de } \\
\text { los candidatos }\end{array}$ & $6^{\prime}$ & $\begin{array}{l}21 \mathrm{~h} 14 \\
\text { '20"' }\end{array}$ & $\begin{array}{ll}\text { Albert } & \left(2^{\prime}\right) \\
\text { Iglesias } & \left(2^{\prime}\right) \\
\text { Pedro } & \left(2^{\prime}\right) \\
\end{array}$ \\
\hline 7 & \begin{tabular}{|l|}
$21 \mathrm{~h} 14$ \\
'20"'
\end{tabular} & $\begin{array}{l}\text { Debate abierto entre } \\
\text { candidatos }\end{array}$ & 9'30" & $\begin{array}{l}21 \mathrm{~h} 23 \\
\text { '50"' }\end{array}$ & \\
\hline 8 & \begin{tabular}{|l|}
$21 \mathrm{~h} 23$ \\
50 '”
\end{tabular} & Preguntas del público & $5^{\prime}$ & $\begin{array}{l}21 \mathrm{~h} 28 \\
\text { '50"' }\end{array}$ & $\begin{array}{l}\text { Albert, Iglesias, } \\
\text { Pedro }\end{array}$ \\
\hline 9 & \begin{tabular}{|l|}
$21 \mathrm{~h} 28$ \\
'50"'
\end{tabular} & $\begin{array}{l}\text { RAFAGA DE } \\
\text { PUBLICIDAD IN/OUT + } \\
\text { CATCH PLATO }\end{array}$ & 3'30" & $\begin{array}{l}21 \mathrm{~h} 32 \\
\text { '20"' }\end{array}$ & VTR PUBLI 3 \\
\hline 10 & $\begin{array}{l}21 \mathrm{~h} 32 \\
20 \text { "' }\end{array}$ & $\begin{array}{c}\text { SEGUNDO BLOQUE } \\
\text { Política Social y } \\
\text { Estado del Bienestar }\end{array}$ & $30 "$ & $\begin{array}{c}21 \mathrm{~h} \\
32^{\prime} 50^{\prime \prime}\end{array}$ & Entra rótulo bloque \\
\hline
\end{tabular}

Fig 4. Escaleta del cara a cara organizado por El País

Fuente: PSOE, material de campaña

El mitin hoy se diseña como cualquier otro producto: estrategias, objetivos, acciones, "target", meta, acciones, promoción.... son hoy el camino por recorrer. Los equipos recurren a agencias especializadas que les ayuden a alcanzar el objetivo final. Para ilustrarlo tomaremos como ejemplo el mitin del PSOE previo a la campaña electoral de 2008 en Vista Alegre, Madrid: 


\begin{tabular}{|c|c|c|c|}
\hline Objetivo & Meta & Acciones & Operativo \\
\hline $\begin{array}{l}\text { Mostrar la capacidad de } \\
\text { movilización del } \\
\text { partido en torno a su } \\
\text { Candidato } \\
\text { Mostrar el } \\
\text { apoyo total del conjunto } \\
\text { de los dirigentes del } \\
\text { partido al Candidato }\end{array}$ & $\begin{array}{l}\text { Con la presencia de todos los } \\
\text { Presidentes de CCAA gobernadas } \\
\text { y } \\
\text { Con la totalidad de alcaldes } \\
\text { socialistas de las grandes } \\
\text { ciudades. }\end{array}$ & $\begin{array}{l}\text { 1. un mes antes de la fecha del } \\
\text { acto: dispositivo de movilización } \\
\text { de militantes y simpatizantes en } \\
\text { toda España } \\
\text { 2. se fijaron objetivos de autobuses } \\
\text { a las estructuras provinciales } \\
\text { 3. convocatoria personalizada a } \\
\text { todos los responsables locales del } \\
\text { partido en cada uno de los } \\
\text { municipios del país } \\
\text { 4. gestión antes las secretarías de } \\
\text { todos los dirigentes y coordinación } \\
\text { de agendas }\end{array}$ & $\begin{array}{l}\text { 1. durante ese mes } 5 \text { personas, } \\
\text { en la sede electoral: } \\
\text { seguimiento diario con cada } \\
\text { uno de los responsables } \\
\text { provinciales y locales del } \\
\text { Partido } \\
\text { 2. días previos al acto: intensa } \\
\text { campaña publicitaria en radio } \\
\text { 3. acciones de marketing } \\
\text { directo en } 350.000 \text { domicilios } \\
\text { de los distritos Madrid } \\
\text { próximos al recinto (buzoneo y } \\
\text { reparto directo }\end{array}$ \\
\hline $\begin{array}{l}\text { Poner en valor la } \\
\text { gestión del Gobierno } \\
\text { en el periodo } 04 / 08\end{array}$ & $\begin{array}{l}\text { Con la presencia de la totalidad de } \\
\text { los miembros del Gobierno de } \\
\text { España en este acto. }\end{array}$ & $\begin{array}{l}\text { Gestión con jefes de gabinetes y } \\
\text { secretarías de todos los miembros } \\
\text { del gobierno y coordinación de } \\
\text { agendas }\end{array}$ & \\
\hline $\begin{array}{l}\text { Visualizar las } \\
\text { propuestas de futuro } \\
\text { para la siguiente } \\
\text { legislatura. }\end{array}$ & $\begin{array}{l}\text { Con la presencia de la totalidad de } \\
\text { los miembros del Gobierno de } \\
\text { España en este acto. }\end{array}$ & $\begin{array}{l}\text { Gestión con jefes de gabinetes y } \\
\text { secretarías de todos los miembros } \\
\text { del gobierno y coordinación de } \\
\text { agendas }\end{array}$ & \\
\hline
\end{tabular}

Fig 5. Objetivos de un mitin

Fuente: PSOE, material de campaña

Los grandes mítines pasan a ser "eventos". Polideportivos, plazas de toros y espacios polivalentes de grandes dimensiones se convierten en contenedores diseñados al milímetro para envolver al candidato como perfecto producto acabado.

\begin{tabular}{|c|c|c|}
\hline $\begin{array}{l}\text { a) Puesta en escena } \\
\text {-el público ocuparía la totalidad del recinto y, } \\
\text { por tanto, los } 360^{\circ} \text { del espacio de graderío se } \\
\text { ocuparían totalmente }\end{array}$ & $\begin{array}{l}\text { - el escenario ocupa una posición central de } \\
\text { forma que los oradores principales aparezcan } \\
\text { rodeados y "arropados" por público } \\
\text {-los elementos decorativos y técnicos deben } \\
\text { integrarse en el espacio permitiendo que la } \\
\text { visibilidad del público quede afectada } \\
\text {-la utilización de las posibilidades que } \\
\text { ofrecen los nuevos soportes audiovisuales } \\
\text { debe reforzar la puesta en escena con la } \\
\text { utilización de formatos diferentes de pantallas }\end{array}$ & \\
\hline a) La escaleta del acto & $\begin{array}{l}\text { Combinación de elementos festivos con } \\
\text { espacios para los discursos y los mensajes: } \\
\text { 1) discurso principal del candidato } \\
\text { 2) apoyo del Partido: intervención de algún } \\
\text { miembro } \\
\text { 3) para visualizar la labor del Gobierno y } \\
\text { presentar al equipo: intervenciones de los } \\
\text { dos Vicepresidentes del Gobierno }\end{array}$ & $\begin{array}{l}\text {-una batucada al inicio del acto } \\
\text {-un espectáculo de Els Comediants cuyo } \\
\text { contenido respondía a una serie de mensajes } \\
\text {-vídeos intercalados en diferentes momentos } \\
\text { del acto como elementos de "ruptura" entre } \\
\text { bloques }\end{array}$ \\
\hline
\end{tabular}

Fig. 6 Guion de un mitin

Fuente: PSOE, material de campaña 
Los equipos de campaña preparan guiones en los que, hábilmente, van intercalando intervenciones de los líderes -de menor a mayor relevancia, pues el protocolo también es una herramienta a tener en cuenta- con actuaciones musicales y otros entretenimientos que convierten al mitin político en una "atracción" pública y gratuita, donde los simpatizantes pueden ir a pasar un rato. Colores y música son fundamentales, como lo son toda nueva tecnología que convierta el espacio en un escaparate de ofertas.

\begin{tabular}{|c|c|c|}
\hline Objetivo & Acción & Operativo \\
\hline $\begin{array}{l}\text { Invitados especiales } \\
\text { a)invitados directos: } \\
\text {-a la Dirección del PSOE a nivel nacional } \\
\text {-al Gobierno de España (Min y SE } \text { SE }^{\circ} \\
\text {-Presidentes CCAA, Secretarios Generales } \\
\text { del PSOE en las CCAA } \\
\text {-Alcaldes (ciudades de más de } 50.000 \text { hab. } \\
\text { b)invitados Sociedad Civil: } \\
\text {-ONG,s } \\
\text {-Sindicatos } \\
\text {-Asociaciones Empresariales }\end{array}$ & -remitir invitación personal a todos & $\begin{array}{l}\text { Equipo de seis personas: } \\
\text { - recibir confirmaciones } \\
\text { - atender las demandas de información: detalles del } \\
\text { acto, accesos, aparcamiento de vehículos, etc. } \\
\text { - elaborar lista de confirmaciones }\end{array}$ \\
\hline b) Recepción y atención a los invitados & $\begin{array}{l}\text {-montar un acceso específico y } \\
\text { diferenciado del público en general } \\
\text {-montar un acceso restringido (por } \\
\text { razones de seguridad para algunos } \\
\text { invitados) } \\
\text {-disponer un equipo de "wellcome" en } \\
\text { este segundo acceso: recibir y } \\
\text { acompañar hasta zona de recepción:. } \\
\text { - acompañar a asientos reservados } \\
\text { (primeras filas frente al escenario } \\
\text { principal) }\end{array}$ & $\begin{array}{l}\text { Para atender a todos estos invitados: un equipo de } 20 \\
\text { personas repartidas en tres zonas: } \\
\text { 1) Área de Accesos: Equipo Wellcome } \\
\text { 2) Área de Recepción y Espera: Equipo de asistencia. } \\
\text { 3) Interior del Recinto: Equipo de acomodación }\end{array}$ \\
\hline c) Un protocolo singular & $\begin{array}{l}\text { Los partidos se rigen por normativas y } \\
\text { criterios internos propios }\end{array}$ & $\begin{array}{l}\text { El responsable de protocolo ordenará las primeras } \\
\text { filas frente al escenario principal con cartelería } \\
\text { nominal en las primeras filas y cartelería } \\
\text { departamental en las posteriores }\end{array}$ \\
\hline
\end{tabular}

Fig. 7 Protocolo de un mitin

Fuente: PSOE, material de campaña

Las producciones de estos mítines son complejas. Hay que contar con especialistas: técnicos de sonido, iluminadores, escenógrafos, informáticos, carpinteros ocupan durante horas los recintos previstos para los mítines. A ello se suma la labor de organización propia de los grandes eventos: control de accesos, ordenación de espacios, reservas de aparcamiento para coches, acreditaciones para los medios de comunicación, espacios VIP para las cúpulas de los partidos, catering, control de flujos o seguridad por enumerar algunos.

\section{CONCLUSIONES}

A la vista del material analizado queda claro que las campañas electorales hoy son una presentación al público de un producto final destinado a ser el inquilino de la Moncloa. Partiendo de las primeras, en las que primaba el mensaje político que se quería transmitir al votante, y en las que simplemente se buscaba la manera de hacerlo llegar de una forma rápida a cuanto más ciudadano mejor, a las actuales, media un mundo. La sociedad española ha ido madurando políticamente y, como consecuencia, es más escéptica y antes de decidirse por una opción política "busca y compara" como ya hace en cualquier otro ámbito de su vida. 
Así, los partidos hoy se marcan el objetivo "presidente" y desarrollan estrategias de venta apoyándose en disciplinas y especialidades -marketing, marketing político, comunicación política, organización de actos, producción de eventos, protocolo- que se han desarrollando y profesionalizado en estos últimos años, para elaborar un producto con una meta: que el votante compre un determinado candidato. Se inicia así el ciclo habitual creación, diseño, elaboración, promoción- para "colocar" la pieza en manos del comprador.

Por ello necesitan exponerlo como tal en estantería de tienda o de supermercado. Eso exige desde un lanzamiento -presentación de la campaña electoral- hasta eventos lúdicos que lo acerquen al votante-comprador, -los mítines-, pasando por campañas publicitarias en los medios de comunicación -programas de entretenimiento o "late night"- y debates comparativas de marcas-.

El candidato no es ajeno al proceso y se somete disciplinado a las órdenes de sus jefes de campaña y asesores. Sabe, que son ceremonias previas a la meta anhelada.

\section{BIBLIOGRAFÍA}

\section{BIBLIOGRAFÍA}

MARTÍNEZ, J. (1998): Historia de España. Siglo XX (1939-1996). Madrid. Cátedra.

PRESTON, P. (2003): Juan Carlos, el rey de un pueblo (volumen II). Hospitalet. ABC, S.L.

DEL REY MORATÓ, Javier (1989): La comunicación política. Madrid. Eudema.

SANTIAGO BARNÉS, J (2007): El político nace o se hace. Salamanca. Amarú Ediciones.

WERT, J.I. (año): La campaña electoral de octubre de 1982: el camino del cambio. Revista REIS, nㄴ28. Pp.69.

\section{WEBGRAFÍA}

ARDANAZ, N. (1989): Los discursos políticos televisivos durante la transición española. Consultado en: http://www.publicacions.ub.es/bibliotecadigital/cinema/filmhistoria/Art.Ardanaz.pdf

EL PAIS (24 Jun 1977): Los partidos realizarán su propia información en RTVE, Consultado en: http://elpais.com/diario/1977/05/24/espana/233272813 850215.html

EL PAIS (2 Feb 1979): El Ministerio de Cultura prohíbe los mítines en los polideportivos, Consultado en: http://elpais.com/diario/1979/02/02/espana/286758013 850215.html 
EL PAIS (3 Feb 1979): Numerosos mítines, sin grandes concentraciones, Consultado en: http://elpais.com/diario/1979/02/03/espana/286844419 850215.html

EL PAÍS (21 Nov 2015): El primer debate digital de la democracia, el lunes día 30, Consultado en: http://politica.elpais.com/politica/2015/11/06/actualidad/1446837878 714795.html

HUFFINGTON POST (13 Dic 2015): Debates en España: pocos y de dos en dos, Consultado en: http://www.huffingtonpost.es/2015/10/13/debates-elecciones-espana n 8286192.html

EL PAÍS ( 14 Dic 2015): Cinco debates cara a cara en once elecciones generales, Consultado en: http://politica.elpais.com/politica/2015/11/26/actualidad/1448533075 331524.html 
